Pacific Journal of Mathematics

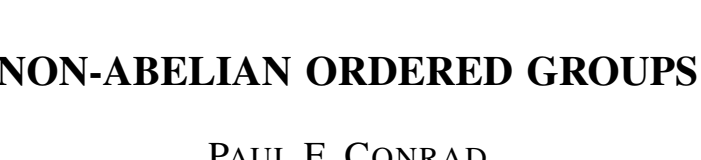




\section{NON-ABELIAN ORDERED GROUPS}

\section{PAUL CONRAD}

1. Introduction. In this paper we prove some theorems about nonabelian o-groups, and give some methods of constructing such groups. Most of the literature on o-groups is concerned with abelian o-groups, and the examples in print of non-abelian o-groups are few. Iwasawa [8] proves that any free group can be ordered, and he also gives some additional examples of o-groups. Vinogradov [15] shows that the free product of two o-groups $A$ and $B$ can be ordered so as to preserve the given orders. Chehata [1] gives an example of an o-group that is simple. [3] and [11] contain examples of o-groups. Most of the theorems in this paper give methods for constructing o-groups. For example, in $\S 3$ we study the o-automorphisms of an o-group $G$. For every group $A$ of o-automorphisms of $G$ that can be ordered we can construct a new o-group $H$ that contains $A$ and $G . H$ is the natural splitting extension of $G$ by $A$. In $\S 5$ the relationship between central extensions and bilinear mappings is exploited. It is shown that any skew-symmetric real matrix can be used to construct o-groups. In $\S 6$ some o-groups of rank 2 are constructed. In $\S 4$ a study is made of the ordered extensions of a subgroup of the reals. One of the main results is a necessary and sufficient condition for such an extension to split. The principal tool used throughout is the extension theory of Schreier [14].

2. Notation and Terminology. The notation of [3] is used throughout. In particular, the notation and results from $\S 2$ [3, pp. 517-518] are used repeatedly. Unless otherwise stated the group operation will always be addition and 0 will denote a group identity. $N$ and $N^{\prime}$ are o-groups with elements $a, b, c, \cdots$ and $a^{\prime}, b^{\prime}, c^{\prime}, \cdots$ respectively. $G$ is a normal o-extension of $N$ by $N^{\prime}$. We identify $G$ with its representation $G^{\prime}=$ $N^{\prime} \times N$, where

$$
\left(a^{\prime}, a\right)+\left(b^{\prime}, b\right)=\left(a^{\prime}+b^{\prime}, f\left(a^{\prime}, b^{\prime}\right)+a r\left(b^{\prime}\right)+b\right)
$$

and $\left(a^{\prime}, a\right)$ is positive if $a^{\prime}>0$ or $a^{\prime}=0$ and $a>0$. See [3] for the properties of the factor mapping $f$ and the representative function $r$.

$\theta$ will always denote a trivial homomorphism of a group onto the identity element of some other group. For an o-group $H$, let $A(H)$ be the group of all o-automorphisms of $H$. For an abelian o-group $K$, let $D(K)$ be the $d$-closure or completion of $K$. In particular, $D(K)$ is a vector space over the rationals and there is a natural extension of the order

Received August 25, 1958. This work was supported by a grant from the National Science Foundation. 
of $K$ to an order of $D(K)$. Finally let $\mathbf{R}$ be the additive group of all real numbers, $\mathbf{P}$ be the multiplicative group of all positive real numbers, $R$ be the additive group of all rational numbers, $\mathbf{P}$ be the multiplicative group of all positive rational numbers, and $I$ be the additive group of integers-all with their natural order.

3. Order preserving automorphisms of G. If $H$ is an o-group and $A$ is a group of o-automorphisms of $H$ that can be ordered, then the group $H^{\prime}=A \times H$, where $(\alpha, a)+(\beta, b)=(\alpha \beta, a \beta+b)$ for $\alpha, \beta$ in $A$ and $a, b$ in $H$, can be ordered. Simply define $(\alpha, a)$ positive if $\alpha$ is positive in $A$ or $\alpha$ is the identity and $a$ is positive in $H$. Then clearly $H^{\prime}$ is a splitting o-extension of $H$ by $A$. Thus if $A$ contains more than one element, then $H^{\prime}$ is a non-abelian o-group. If $A$ is the group of all o-automorphisms of $H$, then $H^{\prime}$ is called the o-holomorph of $H$. In [5] it has been shown that a certain class of o-groups with well ordered rank have ordered o-holomorphs. In this section we investigate the o-automorphisms of $G$.

Let $\pi$ be an o-automorphism of $G$ for which $(0 \times N) \pi=0 \times N$. and let $\mathscr{A}$ be the group of all these o-automorphisms. If $G$ has well ordered rank or if $N^{\prime}$ or $N$ has finite rank, then $\mathscr{A}=A(G)$. For $\left(\alpha^{\prime}, a\right)$ and $\left(b^{\prime}, b\right)$ in $G$ we have

$$
\begin{gathered}
\left(a^{\prime}, a\right) \pi=\left[\left(a^{\prime}, 0\right)+(0, a)\right] \pi=\left(a^{\prime}, 0\right) \pi+(0, a) \pi \\
=\left(a^{\prime} \alpha, a^{\prime} \beta\right)+(0, a \gamma)=\left(a^{\prime} \alpha, a^{\prime} \beta+a \gamma\right),
\end{gathered}
$$

where

$$
\begin{gathered}
0 \beta=0 . \\
{\left[\left(a^{\prime}, a\right)+\left(b^{\prime}, b\right)\right] \pi=\left(a^{\prime}+b^{\prime}, f\left(a^{\prime}, b^{\prime}\right)+a r\left(b^{\prime}\right)+b\right) \pi} \\
=\left(\left(a^{\prime}+b^{\prime}\right) \alpha,\left(a^{\prime}+b^{\prime}\right) \beta+\left(f\left(a^{\prime}, b^{\prime}\right)+a r\left(b^{\prime}\right)+b\right) \gamma\right) . \\
\left(a^{\prime}, a\right) \pi+\left(b^{\prime}, b\right) \pi=\left(a^{\prime} \alpha, a^{\prime} \beta+a \gamma\right)+\left(b^{\prime} \alpha, b^{\prime} \beta+b \gamma\right) \\
=\left(a^{\prime} \alpha+b^{\prime} \alpha, f\left(a^{\prime} \alpha, b^{\prime} \alpha\right)+\left(a^{\prime} \beta+a \gamma\right) r\left(b^{\prime} \alpha\right)+b^{\prime} \beta+b \gamma\right) .
\end{gathered}
$$

Thus $\alpha \in A\left(N^{\prime}\right)$ and

$$
\begin{aligned}
& \left(a^{\prime}+b^{\prime}\right) \beta+\left(f\left(a^{\prime}, b^{\prime}\right)+a r\left(b^{\prime}\right)+b\right) \gamma \\
& \quad=f\left(a^{\prime} \alpha, b^{\prime} \alpha\right)+\left(a^{\prime} \beta+a \gamma\right) r\left(b^{\prime} \alpha\right)+b^{\prime} \beta+b \gamma .
\end{aligned}
$$

When $a^{\prime}=b^{\prime}=0$ this reduces to $(a+b) r=a r+b r$. Thus $r \in A(N)$. The following two equations are the result of letting $a^{\prime}=b=0(a=b=0)$.

$$
\begin{gathered}
b^{\prime} \beta+\operatorname{ar}\left(b^{\prime}\right) \gamma=\operatorname{arr}\left(b^{\prime} \alpha\right)+b^{\prime} \beta \\
\left(a^{\prime}+b^{\prime}\right) \beta+f\left(a^{\prime}, b^{\prime}\right) \gamma=f\left(a^{\prime} \alpha, b^{\prime} \alpha\right)+a^{\prime} \beta r\left(b^{\prime} \alpha\right)+b^{\prime} \beta .
\end{gathered}
$$

Conversely suppose that $\alpha \in A\left(N^{\prime}\right), \gamma \in A(N), \beta: N^{\prime} \rightarrow N$, and (1), (2), (3) 
are satisfied. For $\left(a^{\prime} a\right)$ in $G$ define $\left(a^{\prime}, a\right) \pi=\left(a^{\prime} \alpha, a^{\prime} \beta+a \gamma\right)$. Then by straightforward computation it follows that $\pi \in \mathscr{O}$.

For mappings $u$ and $v$ of $N^{\prime}$ into $N$ and $a^{\prime} \in N^{\prime}$ we define $a^{\prime}(u+v)=$ $a^{\prime} u+a^{\prime} v$. Then each $\pi \in \mathscr{A}$ has a matrix representation

$$
\left[\begin{array}{l}
\alpha \beta \\
\theta \gamma
\end{array}\right]
$$

where $\theta$ is the trivial homomorphism of $N$, into $N^{\prime}$, and the mapping of $\pi$ onto its matrix representation is an isomorphism of $\mathscr{A}$ onto

$$
\left\{\left[\begin{array}{l}
\alpha \beta \\
\theta \gamma
\end{array}\right]: \alpha \in A(N), \gamma \in A\left(N^{\prime}\right), \beta: N^{\prime} \rightarrow N \text {, and (1), (2), (3) are satisfied }\right\} .
$$

For, let $\pi=(\alpha, \beta, \gamma)$ and $\bar{\pi}=(\bar{\alpha}, \bar{\beta}, \bar{\gamma})$, then

$$
\begin{aligned}
\left(\alpha^{\prime}, \bar{r} \alpha\right) \vec{\tau} \pi & =\left(a^{\prime} \bar{\alpha}, a^{\prime} \bar{\beta}+a \bar{\gamma}\right) \pi=\left(a^{\prime} \bar{\alpha} \alpha, \alpha^{\prime} \bar{\alpha} \beta+\left(a^{\prime} \bar{\beta}+a \bar{r}\right) \gamma\right) \\
& =\left(a^{\prime} \bar{\alpha} \alpha, a^{\prime}(\bar{\alpha} \beta+\bar{\beta} \gamma)+a \bar{\gamma} \gamma\right)
\end{aligned}
$$

and

$$
\left[\begin{array}{l}
\bar{\alpha} \bar{\beta} \\
\theta \bar{\gamma}
\end{array}\right]\left[\begin{array}{l}
\alpha \beta \\
\theta \gamma
\end{array}\right]=\left[\begin{array}{cc}
\bar{\alpha} \alpha & \bar{\alpha} \beta+\bar{\beta} \gamma \\
\theta & \bar{\gamma} \gamma
\end{array}\right]
$$

We shall frequently identity the elements of $\mathscr{A}$ with their matrix representation. Let $\mathscr{S}$ be the set of all $\beta: N^{\prime} \rightarrow N$ that satisfy (1), (2), (3) when $\alpha$ and $\gamma$ are the identity automorphisms of $N^{\prime}$ and $N$ respectively.

LEMMA 3.1. T) is an additive group that can be ordered.

Proof. From the matrix representation of $\mathscr{A}$ it follows that $\mathscr{S}$ is a group. Well order the elements of $N^{\prime}$ and define $\beta \in C$. if $\beta \neq \theta$ and $a^{\prime} \beta>0$, where $a^{\prime}$ is the first element in the well ordering for which $\alpha^{\prime} \beta \neq 0$. It is easy to check that this definition orders $\mathscr{B}$.

COROLlaRY I. The group of all mappings of a set onto an o-group can be ordered.

CoRollary II. The group of all o-automorphisms of $G$ that induce the identity automorphism on $G /(0 \times N)$ and on $0 \times N$ can be ordered.

Now suppose that $\mathscr{B}, A\left(N^{\prime}\right)$ and $A(N)$ are o-groups and let

$$
\pi=\left[\begin{array}{l}
\alpha \beta \\
\theta \gamma
\end{array}\right] \quad \bar{\pi}=\left[\begin{array}{l}
\bar{\alpha} \bar{\beta} \\
\theta \bar{\gamma}
\end{array}\right]
$$

be elements of $\mathscr{A}$. Then

(5) $\pi^{-1}=\left[\begin{array}{c}\alpha^{-1}-\alpha^{-1} \beta \gamma^{-1} \\ \theta \\ \gamma^{-1}\end{array}\right] \quad \pi^{-1} \bar{\pi} \pi=\left[\begin{array}{cc}\alpha^{-1} \bar{\alpha} \alpha & \alpha^{-1}(\bar{\alpha} \beta+\bar{\beta} \gamma) \underset{\theta}{\gamma^{-1} \bar{\gamma} \gamma} \alpha^{-1} \beta \gamma^{-1} \bar{\gamma} \gamma\end{array}\right]$ 
Definition 3.1. $\pi$ is positive if $\alpha$ is positive in $A\left(N^{\prime}\right)$ or $\alpha$ is the identity and $\gamma$ is positive in $A(N)$ or $\alpha$ ond $\gamma$ are identity automorphisms and $\beta$ is positive in $\mathscr{R}$.

Let $\mathscr{P}$ be the set of all positive elements in $\mathscr{A}$. It follows from (4) that $\mathscr{P}$ is closed with respect to multiplication. It follows from the first part of (5) that for each $\pi \in \mathscr{A}$, either $\pi$ is the identity or $\pi \in \mathscr{P}$ or $\pi^{-1} \in \mathscr{P}$. Unfortunately $\mathscr{P}$ is not in general normal. For suppose that $\bar{\pi} \in \mathscr{P}$, then if $\bar{\alpha}$ is positive or $\bar{\gamma}$ is positive, then $\pi^{-1} \bar{\pi} \pi$ is positive. Finally assume that $\bar{\alpha}$ and $\bar{\gamma}$ are identity automorphisms, then

$$
\pi^{-1} \bar{\pi} \pi=\left[\begin{array}{cc}
\phi^{\prime} & \alpha^{-1}(\beta+\bar{\beta} \gamma-\beta) \\
\theta & \phi
\end{array}\right],
$$

where $\phi^{\prime}(\phi)$ is the identity of $A\left(N^{\prime}\right)(A(N))$. Thus our definition orders $\mathscr{A}$ if and only if $\alpha^{-1}(\beta+\bar{\beta} \gamma-\beta)=\alpha^{-1} \beta+\alpha^{-1} \bar{\beta} \gamma-\alpha^{-1} \beta$ is positive. If we use the ordering of $\mathscr{S}$ defined in the proof of Lemma 3.1, then it suffices to show that $\alpha^{\prime} \alpha^{-1} \bar{\beta}>0$, where $\alpha^{\prime}$ is the first element in the well ordering of $N^{\prime}$ such that $\alpha^{\prime} \alpha^{-1} \bar{\beta} \neq 0$.

THEOREm 3.1. If $A(N)$ can be ordered, then the group of all o-automorphisms $\pi$ of $G$ such that $(0 \times N) \pi=0 \times N$ and $\pi$ induces the identity automorphism on $G /(0 \times N)$ can be ordered.

We next consider the special cases where $G$ is a central extension of $N$ or where $G$ is a splitting extension of $N$. First assume that $N$ (actually $0 \times N$ ) is in the center of $G$. Then $r=\theta$ and $N$ is abelian. In particular, (1), (2), (3) reduce to

$$
\left(a^{\prime}+b^{\prime}\right) \beta+f\left(a^{\prime}, b^{\prime}\right) \gamma=f\left(a^{\prime} \alpha, b^{\prime} \alpha\right)+a^{\prime} \beta+b^{\prime} \beta
$$

and $0 \beta=0$. Thus $\mathscr{B}$ is the torsion free abelian group $H\left(N^{\prime}, N\right)$ of all homomorphisms of $N^{\prime}$ into $N$. Let $\Gamma$ be the set of all ordered pairs of convex subgroups $N^{\prime \gamma}, N^{\prime}{ }_{\gamma}$ of $N^{\prime}$ such that $N^{\prime \gamma}$ covers $N^{\prime}{ }_{\gamma}$.

THEOREM 3.2. Suppose that $G$ is a central extension of $N, A(N)$ can be ordered, $\Gamma$ is well ordered, and for each pair $\alpha \in A\left(N^{\prime}\right)$ and $\gamma \in \Gamma$ there exists a pair of positive integers $m$ and $n$ such that ng $\alpha \equiv m g$ modulo $N^{\prime}{ }_{\gamma}$ for all $g \in N^{\prime \gamma}$. Then $A\left(N^{\prime}\right)$ and $\mathscr{A}$ can be ordered.

Proof. By the theorem in [5], $A\left(N^{\prime}\right)$ can be ordered. As in the proof of Theorem 3 [4 p. 388] we well order the elements of $N^{\prime}$ so that

$$
\underset{0 \rightarrow \frac{g_{11} \rightarrow g_{12}}{N^{\prime 1}}}{0 .} \frac{g_{21} \rightarrow g_{22} \rightarrow \cdots}{N^{\prime 2} \backslash N^{\prime}{ }_{2}} \ldots \frac{g_{\omega 1} \rightarrow g_{\omega 2} \rightarrow \cdots}{N^{\prime \omega} \backslash N^{\prime}{ }_{\omega}} \ldots
$$

For each $\theta \neq \beta \in \mathscr{S}$ there exists a least element $L(\beta)$ in this well 
ordering such that $L(\beta) \beta \neq 0$. Define $\beta$ positive if $L(\beta) \beta>0$. As before this orders $\mathscr{B}$. Thus to complete the proof it suffices to show that if $\beta$ is positive, then $\alpha \beta$ is positive for all $\alpha \in A\left(N^{\prime}\right)$. Let $g \in N^{\prime \gamma} / N^{\prime}{ }_{\gamma}$. Then there exist positive integers $m$ and $n$ such that $n(g \alpha)=m g+d$, where $d \in N^{\prime}{ }_{\gamma}$, hence $d \rightarrow g$. If $g \rightarrow L(\beta)$, then

$$
n(g \alpha \beta)=(m g+d) \beta=m(g \beta)+d \beta=0 .
$$

Thus $g \alpha \beta=0$. If $g=L(\beta)$, then

$$
n(L(\beta) \alpha \beta)=(m L(\beta)+d) \beta=m(L(\beta) \beta)+d \beta=m(L(\beta) \beta)>0 .
$$

Thus $L(\beta) \alpha \beta>0$.

Corollary. If $N$ is in the center of $G, A(N)$ can be ordered and $N^{\prime}=R$, then $A(G)$ can be ordered.

One should be careful not to place too many restrictions on $G$. For $A(G)$ may become trivial (consist of the identity only). de Groot [6] has shown that exist $2^{c}$ non-isomorphic archimedean o-groups that admit only the identity automorphism. Suppose that $G$ admits no proper o-automorphism and that $N^{\prime}$ and $N$ are non-trivial. Then, since an inner automorphism is an o-automorphism, $G$ is abelian. Hence $N$ is in the center of $G$. Thus in order to construct a non-archimedean o-group that admits only the trivial o-automorphism, it suffices to find non-trivial subgroups $N^{\prime}$ and $N$ of $\mathbf{R}$ such that neither admit proper o-automorphisms and the only homomorphism of $N^{\prime}$ into $N$ is $\theta$. Then $G=N^{\prime} \oplus N$ will do. One such pair is

$$
N=I \text { and } N^{\prime}=\left\{m / 2^{n}: m, n \in I\right\} e+\left\{p / 3^{q}: p, q \in I\right\},
$$

where $e$ is trancendental.

For the remainder of this section assume that $G$ is a splitting extension of $N$ by $N^{\prime}$ and that $N \subseteq \mathbf{R}$. Without loss of generality $f\left(a^{\prime}, b^{\prime}\right)=0$ for all $a^{\prime}, b^{\prime}$ in $N^{\prime}$ and $A(N) \subseteq \mathbf{P}$. Thus $r\left(b^{\prime}\right), \gamma \in \mathbf{P}$, and $\operatorname{ar}\left(b^{\prime}\right)$, ar represent ordinary multiplication, where $a \in N, b^{\prime} \in N^{\prime}$ and $\gamma \in A(N)$. In particular, (2) and (3) reduce to

$$
\begin{aligned}
r\left(b^{\prime}\right) & =r\left(b^{\prime} \alpha\right), \text { and } \\
\left(a^{\prime}+b^{\prime}\right) \beta & =a^{\prime} \beta r\left(b^{\prime}\right)+b^{\prime} \beta .
\end{aligned}
$$

Pick an element $k \in N$ and define $x^{\prime} \beta=k\left(r\left(x^{\prime}\right)-1\right)$ for all $x^{\prime} \in N^{\prime}$. $a^{\prime} \beta r\left(b^{\prime}\right)+b^{\prime} \beta=k\left(r\left(a^{\prime}\right)-1\right) r\left(b^{\prime}\right)+k\left(r\left(b^{\prime}\right)-1\right)=k\left(r\left(a^{\prime}\right) r\left(b^{\prime}\right)-1\right)=k\left(r\left(a^{\prime}+\right.\right.$ $\left.\left.b^{\prime}\right)-1\right)=\left(a^{\prime}+b^{\prime}\right) \beta$. Thus $\beta \in \mathscr{B}$. Suppose that there exists an element $a^{\prime}$ in the center of $N^{\prime}$ such that $r\left(a^{\prime}\right) \neq 1$. Let $x^{\prime}$ be any other 
element of $N^{\prime}$, and let $\beta \in \mathscr{S}$. Then $x^{\prime} \beta r\left(\alpha^{\prime}\right)+\alpha^{\prime} \beta=\left(x^{\prime}+\alpha^{\prime}\right) \beta=$ $\left(\alpha^{\prime}+x^{\prime}\right) \beta=a^{\prime} \beta r\left(x^{\prime}\right)+x^{\prime} \beta$. Thus $x^{\prime} \beta\left(r\left(\alpha^{\prime}\right)-1\right)=a^{\prime} \beta\left(r\left(x^{\prime}\right)-1\right)$ or

$$
x^{\prime} \beta=\left[\begin{array}{c}
\alpha^{\prime} \beta \\
r\left(\alpha^{\prime}\right)-1
\end{array}\right]\left[r\left(x^{\prime}\right)-1\right] .
$$

Therefore $\beta$ is determined by $a^{\prime} \beta$.

LEMMA 3.2. If there exists an element $\alpha^{\prime}$ in the center of $N^{\prime}$ such that $r\left(a^{\prime}\right) \neq 1$, then $\mathscr{S}$ is isomorphic to a subgroup of $\mathbf{R}$ that contains $N$.

Proof. For $\beta \in \mathscr{B}$ we define $\beta \sigma=\left(\alpha^{\prime} \beta\right) /\left(r\left(\alpha^{\prime}\right)-1\right)$. Then

$$
\begin{aligned}
& \left(\beta_{1}+\beta_{2}\right) \sigma=a^{\prime}\left(\beta_{1}+\beta_{2}\right) /\left(r\left(a^{\prime}\right)-1\right)=\left(a^{\prime} \beta_{1}\right) /\left(r\left(a^{\prime}\right)-1\right) \\
& \quad+\left(a^{\prime} \beta_{2}\right) /\left(r\left(a^{\prime}\right)-1\right)=\beta_{1} \sigma+\beta_{2} \sigma .
\end{aligned}
$$

If $0=\beta \sigma=\left(a^{\prime} \beta\right) /\left(r\left(\alpha^{\prime}\right)-1\right)$, then $\alpha^{\prime} \beta=0$. Thus by (6), $\beta=\theta$. Therefore $\sigma$ is an isomorphism of $\mathscr{B}$ into $\mathbf{R}$, and by the preceding discussion $\mathscr{B} \sigma \supseteq N$.

If $r\left(a^{\prime}\right)<1$, then $1<r\left(a^{\prime}\right)^{-1}=r\left(-a^{\prime}\right)$. Thus we may assume that $r\left(a^{\prime}\right)-1>0$. Define $\beta \in \mathscr{B}$ positive (notation) $\beta>\theta$ ) if $\beta \sigma>0$. Then $\mathscr{B}$ is ordered and $A(N) \subseteq \mathbf{P}$ has a natural order. $\beta \sigma=\left(\alpha^{\prime} \beta\right) /\left(r\left(\alpha^{\prime}\right)-\right.$ 1) $>0$ if and only if $\alpha^{\prime} \beta>0$. Thus $\beta>\theta$ if and only if $\alpha^{\prime} \beta>0$. Suppose that $A\left(N^{\prime}\right)$ is also ordered. Then Definition 3.1 orders $A(G)$ if we can show that $\bar{\beta}>\theta$ implies that $\alpha^{-1}(\beta+\bar{\beta} \gamma-\beta)>\theta$ for all $\bar{\beta} \in \mathscr{B}$, and all $\pi=(\alpha, \beta, \gamma) \in A(G)$. But

$$
\begin{aligned}
& a^{\prime} \alpha^{-1}(\beta+\bar{\beta} \gamma-\beta)=a^{\prime} \alpha^{-1} \bar{\beta} \gamma=\left(\left(a^{\prime} \alpha^{-1}\right) \bar{\beta}\right) \gamma \\
& =\left[\left(\alpha^{\prime} \bar{\beta}\right)\left(r\left(a^{\prime} \alpha^{-1}\right)-1\right) /\left(r\left(\alpha^{\prime}\right)-1\right)\right] \gamma=\alpha^{\prime} \bar{\beta} \gamma .
\end{aligned}
$$

But since $a^{\prime} \bar{\beta}>0$ we have $a^{\prime} \bar{\beta} \gamma>0$.

THEOREM 3.3. If $G$ splits over $N, N \subseteq \mathbf{R}, A\left(N^{\prime}\right)$ can be ordered and there exists an element $a^{\prime}$ in the center of $N^{\prime}$ such that $r\left(a^{\prime}\right) \neq 1$, then $A(G)$ can be ordered.

COROLlaRY. If $H$ is a non-abelian splitting o-extension of a subgroup of $\mathbf{R}$ by a subgroup of $\mathbf{R}$, then $A(H)$ can be ordered.

This is an immediate consequence of the theorem. If $N^{\prime}=\mathbf{R}$, then $\left(2^{\prime}\right)$ is equivalent to $1=r\left(b^{\prime}(\alpha-1)\right)$. Hence either $r=\theta$ or $\alpha=1$. Thus if $N^{\prime}=\mathbf{R}$, then this corollary is an immediate consequence of Theorem 3.1. 
4. Ordered extension of subgroups of $\mathbf{R}$. Throughout this section assume that $N$ is a subgroup of $\mathbf{R}$ and that $N^{\prime}$ is abelian. In particular, $r$ is a homomorphism of $N^{\prime}$ into the group $A(N)$, and without loss of generality $A(N) \subseteq \mathbf{P}$ and $\operatorname{ar}\left(b^{\prime}\right)$ is ordinary multiplication, where $a \in N$ and $b^{\prime} \in N$.

$$
\left(\alpha^{\prime}, a\right)+(0, b)=\left(\alpha^{\prime}, a+b\right) \text { and }(0, b)+\left(a^{\prime}, \alpha\right)=\left(a^{\prime}, b r\left(a^{\prime}\right)+a\right) .
$$

These are equal if and only if $\operatorname{br}\left(a^{\prime}\right)=b$. Thus $G$ is a central extension of $N$ by $N^{\prime}$ if and only if $r=\theta$.

Lemma 4.1. Suppose that $N^{\prime}$ is d-closed. Then there exists a noncentral o-extension of $N$ by $N^{\prime}$ if and only if there exists $1 \neq p \in \mathbf{P}$ such that $p^{s} N=N$ for all $s \in R$.

Proof. First suppose that $G$ is a non-central o-extension of $N$ by $N^{\prime}$. Then $r \neq \theta$. Pick $a^{\prime} \in N^{\prime}$ so that $1 \neq r\left(a^{\prime}\right)=p \in \mathbf{P}$. For each positive integer $n$ there exists $b^{\prime} \in N^{\prime}$ such that $n b^{\prime}=a^{\prime}$. Hence $p=r\left(a^{\prime}\right)=$ $r\left(n b^{\prime}\right)=r\left(b^{\prime}\right)^{n}$. Thus $r\left(b^{\prime}\right)=p^{1 / n}$. For $m \in I$, we have $r\left(m b^{\prime}\right)=r\left(b^{\prime}\right)^{m}=$ $p^{m / n}$. Thus $p^{m / n} N=N$ for all rational numbers $m / n$.

Conversely suppose that there exists $1 \neq p \in \mathbf{P}$ such that $p^{s} N=N$ for all $s \in R$. Pick $0 \neq b^{\prime} \in N^{\prime}$. Then $N^{\prime}=R b^{\prime} \oplus D$, where $R a^{\prime}$ is the one dimensional subspace of $N^{\prime}$ that contains $a^{\prime}$ and $D$ is a subspace of $N^{\prime}$. Each $a^{\prime} \in N^{\prime}$ has a unique representation $a^{\prime}=s b^{\prime}+d$, where $s \in R$ and $d \in D$. Define $q\left(a^{\prime}\right)=p^{s}$. Then $H=N^{\prime} \times N$, where $\left(a^{\prime}, a\right)+$ $\left(b^{\prime}, b\right)=\left(a^{\prime}+b^{\prime}, a q\left(b^{\prime}\right)+b\right)$ is a splitting extension of $N$ by $N^{\prime}$ that is not a central extension.

COROLlaRY. If $N^{\prime}$ is $d$-closed and $N \subseteq R$, then $G$ is a central extension of $N$ by $N^{\prime}$.

Theorem 4.1. Suppose that $r \neq \theta$. Then $G$ splits over $N$ if and only if there exist $a^{\prime} \in N^{\prime}$ and $a \in N$ such that

(a) $r\left(a^{\prime}\right) \neq 1$

(b) $\left[1 /\left(r\left(a^{\prime}\right)-1\right)\right]\left[\alpha\left(r\left(b^{\prime}\right)-1\right)+f\left(a^{\prime}, b^{\prime}\right)-f\left(b^{\prime}, a^{\prime}\right)\right] \in N$ for all $b^{\prime} \in N^{\prime}$.

Proof. First suppose that $G$ splits. Choose a group $H$ of representatives of $G / N$, and pick one element $\left(\alpha^{\prime}, a\right)$ of $H$ such that $r\left(\alpha^{\prime}\right) \neq 1$. Let $\left(b^{\prime}, b\right)$ be any other element of $H$. Then since $H$ is abelian,

$$
\begin{aligned}
& \left(b^{\prime}+a^{\prime}, f\left(b^{\prime}, a^{\prime}\right)+b r\left(a^{\prime}\right)+a\right)=\left(b^{\prime}, b\right)+\left(a^{\prime}, a\right)=\left(a^{\prime}, a\right)+\left(b^{\prime}, b\right) \\
& \quad=\left(a^{\prime}+b^{\prime}, f\left(a^{\prime}, b^{\prime}\right)+\operatorname{ar}\left(b^{\prime}\right)+b\right) .
\end{aligned}
$$

Thus 


$$
b\left(r\left(a^{\prime}\right)-1\right)=a\left(r\left(b^{\prime}\right)-1\right)+f\left(a^{\prime}, b^{\prime}\right)-f\left(b^{\prime}, a^{\prime}\right) .
$$

(b) is satisfied because

$$
\left[1 /\left(r\left(a^{\prime}\right)-1\right)\right]\left[a\left(r\left(b^{\prime}\right)-1\right)+f\left(a^{\prime}, b^{\prime}\right)-f\left(b^{\prime}, a^{\prime}\right)\right]=b .
$$

Note that

$$
H=\left\{\left(b^{\prime},\left[1 /\left(r\left(a^{\prime}\right)-1\right)\right]\left[a\left(r\left(b^{\prime}\right)-1\right)+f\left(a^{\prime}, b^{\prime}\right)-f\left(b^{\prime}, a^{\prime}\right)\right]\right): b^{\prime} \in N^{\prime}\right\} .
$$

Thus $H$ is uniquely determined by $\left(a^{\prime}, a\right)$.

Conversely suppose that $a^{\prime} \in N^{\prime}$ and $a \in N$ satisfy (a) and (b).

Let

$$
S=\left\{\left(b^{\prime}, b\right) \in G:\left(b^{\prime}, b\right)+\left(a^{\prime}, a\right)=\left(a^{\prime}, a\right)+\left(b^{\prime}, b\right)\right\} .
$$

Clearly $S$ is a group. By the above computation it follows that $\left(b^{\prime}, b\right) \in S$ if and only if

$$
b=\left[1 /\left(r\left(a^{\prime}\right)-1\right)\right]\left[a\left(r\left(b^{\prime}\right)-1\right)+f\left(a^{\prime}, b^{\prime}\right)-f\left(b^{\prime}, a^{\prime}\right)\right] .
$$

Thus for each $b^{\prime} \in N^{\prime}$ there is one and only one $\left(b^{\prime}, b\right)$ in $S$. Therefore $S$ is a group of representatives for $G / N$.

The factor mapping $f$ is symmetric (skew-symmetric) if $f\left(a^{\prime}, b^{\prime}\right)=$ $f\left(b^{\prime}, a^{\prime}\right)\left(f\left(a^{\prime}, b^{\prime}\right)=-f\left(b^{\prime}, a^{\prime}\right)\right)$ for all $a^{\prime}, b^{\prime}$ in $N^{\prime}$.

CoRollary I. If $r \neq \theta$ and $f$ is symmetric, then $G$ splits. Moreover $f\left(a^{\prime}, b^{\prime}\right)=0$ for all $a^{\prime}, b^{\prime}$ in $N^{\prime}$.

Proof. Pick $a^{\prime} \in N^{\prime}$ such that $r\left(a^{\prime}\right) \neq 1$ and let $a=0$. Then (a) and (b) are satisfied, hence $G$ splits. Also by the proof of the converse of the theorem, $S=\left\{\left(b^{\prime}, 0\right): b^{\prime} \in N^{\prime}\right\}$ is a group of representatives. Thus $\left(a^{\prime}, 0\right)+\left(b^{\prime}, 0\right)=\left(a^{\prime}+b^{\prime}, f\left(a^{\prime}, b^{\prime}\right)\right) \in S$. Therefore $f\left(a^{\prime}, b^{\prime}\right)=0$

Let $f\left(N^{\prime}, N^{\prime}\right)$ denote the range of $f$.

Corollary II. If there exists an $a^{\prime} \in N^{\prime}$ such that $r\left(a^{\prime}\right) \neq 1$ and $\left[1 /\left(r\left(a^{\prime}\right)-1\right)\right] f\left(N^{\prime}, N^{\prime}\right) \subseteq N$, then $G$ splits.

Proof. Let $a=0$. Then (a) and (b) are satisfied. Moreover, $\left\{\left(b^{\prime},\left[1 /\left(r\left(a^{\prime}\right)-1\right)\right]\left[f\left(a^{\prime}, b^{\prime}\right)-f\left(b^{\prime}, a^{\prime}\right)\right]\right)\right\}$ is a group of representatives.

Corollary III. If $N$ is a field and $r \neq \theta$, then $G$ splits.

Proof. Pick $a^{\prime} \in N^{\prime}$ such that $r\left(a^{\prime}\right) \neq 1$. Since $1 \in N$ and $r\left(a^{\prime}\right) N=$ $N, r\left(a^{\prime}\right) \in N$. Thus $1 /\left(r\left(a^{\prime}\right)-1\right) \in N$ and

$$
\left[1 /\left(r\left(a^{\prime}\right)-1\right)\right] f\left(N^{\prime}, N^{\prime}\right) \subseteq\left[1 /\left(r\left(a^{\prime}\right)-1\right)\right] N=N .
$$

REMARK. Rich [13] proved that if $N \subseteq \mathbf{R}, N^{\prime}=\mathbf{R}$ and $r \neq \theta$, then 
$G$ splits. This is a special case of Corollary III. Corollary III can be stated independently of the representation of $G$ as follows: If $H$ is an o-group, $C$ is a convex subgroup of $H$ that is o-isomorphic to the additive group of a subfield of $\mathbf{R}$, and $H / C$ is abelian, then either $H$ is a splitting extension of $C$ or $H$ is a central extension of $C$.

CoRollary IV. If there exists an $a^{\prime} \in N^{\prime}$ such that $r\left(a^{\prime}\right)=(n+1) / n$ for some positive integer $n$, then $G$ splits.

Proof. $1 /\left(r\left(\alpha^{\prime}\right)-1\right)=n$. Thus $\left[1 /\left(r\left(\alpha^{\prime}\right)-1\right)\right] f\left(N^{\prime}, N^{\prime}\right)=n f\left(N^{\prime}, N^{\prime}\right) \subseteq N$.

Corollary V. If $N$ is $d$-closed and there exists an $a^{\prime} \in N^{\prime}$ such that $1 \neq r\left(a^{\prime}\right)$ is rational, then $G$ splits.

Proof. $1 /\left(r\left(a^{\prime}\right)-1\right)$ is rational, hence $\left[1 /\left(r\left(a^{\prime}\right)-1\right)\right] N \subseteq N$.

By Theorem 3.3 [3, p. 522] there exists an $a$-extension $H$ of $G$ such that the convex subgroup $K$ of $H$ that covers 0 is o-isomorphic to $\mathbf{R}$ and $H / K$ is o-isomorphic to $N^{\prime}$. Thus by Theorem 4.1 either $H$ is a splitting extension of $K$ or $H$ is a central extension of $K$.

REMARK. If $H$ is a splitting o-extension of $K$, then without loss of generality $H=N^{\prime} \times \mathbf{R}$, where $\left(a^{\prime}, a\right)+\left(b^{\prime}, b\right)=\left(a^{\prime}+b^{\prime}, a s\left(b^{\prime}\right)+b\right)$. $s$ is a homomorphism of $N^{\prime}$ into $\mathbf{P}$. For each $x$ in $D(N)$ there exists a positive integer $n$ such that $n x \in N^{\prime}$. Define $t(x)=[s(n x)]^{1 / n}$. Then $t$ is the unique extension of $s$ to a homomorphism of $D\left(N^{\prime}\right)$ into $\mathbf{P}$. $D\left(N^{\prime}\right), \mathbf{R}$ and $t$ determine a splitting o-extension $M$ of $\mathbf{R}$ by $D(N) . M$ is an $a$-extension of $H$ and $M$ is $d$-closed. Thus by Theorem 3.2 [3 p. 519] there exists an $a$-closed a-extension $Q$ of $M$ with each component $o$-isomorphic to R. $Q$ is an $a$-extension of $G$.

A mapping $g$ of $N^{\prime} \times N^{\prime}$ into $N$ is called bilincar if for all $x, y, z$ in $N^{\prime}$

$$
g(x+y, z)=g(x, z)+g(y, z),
$$

and

$$
g(x, y+z)=g(x, y)+g(x, z) .
$$

Yamabe [16] and the Neumanns [12] have shown that if $N=I$, and the cardinality of $N^{\prime}$ is at most $\aleph_{1}$, and $g$ is bilinear and satisfies $g(x, x)=0$ only if $x=0$, then $N^{\prime}$ is a free abelian group. Hughes [7] has classified the groups of class 2 in terms of some special bilinear mappings. Iwasawa gives an example ([8] Example 2, p. 7) of an 0 -group that is determined by a bilinear mapping. For let $N^{\prime}=I \times I$ and $N=I$. Define $g((a, b),(x, y))=$ ay. Then $G=I \times I \times I$, where $(a, b, c)+(x, y, z)=(a+x, b+y, a y+c+z)$, 
and $(a, b, c)$ is positive if $a>0$ or $a=0$ and $b>0$ or $a=b=0$ and $c>0$, is an o-group of rank 3 that is isomorphic with Iwasawa's example. In fact, $G$ is generated by $a=(0,0,1), b=(0,1,0)$ and $c=(1,0,0)$ and has generating relations $a+b=b+a, a+c=c+a$ and $c+b-c=a+b$.

The last example can be generalized because the bilinear form is a product of homomorphisms. For example, let $N$ be the additive group of an ordered ring, and let $\sigma$ and $\tau$ be homomorphisms of $N^{\prime}$ into $N$. For $a^{\prime}, b^{\prime}$ in $N^{\prime}$ define $g\left(a^{\prime}, b^{\prime}\right)=\sigma\left(a^{\prime}\right) \tau\left(b^{\prime}\right)$. Then $H=N^{\prime} \times N$, where $\left(a^{\prime}, a\right)+\left(b^{\prime}, b\right)=\left(a^{\prime}+b^{\prime}, g\left(a^{\prime}, b^{\prime}\right)+a+b\right)$ is a central extension of $N$ by $N^{\prime}$.

Lemma 4.2. If $f$ is bilinsar, then $G$ is a splitting extension of $N$ or $G$ is a central extension of $N$.

Proof. For $x, y, z$ in $N^{\prime}$ we have

$$
\begin{aligned}
f(x, y)+f(x, z)+f(y, z) & =f(x, y+z)+f(y, z)=f(x+y, z)+f(x, y) r(z) \\
& =f(x, z)+f(y, z)+f(x, y) r(z) .
\end{aligned}
$$

Therefore $f(x, y) \equiv f(x, y) r(z)$. Thus either $r(z) \equiv 1$ or $f(x, y) \equiv 0$.

Corollary. If $N$ is abelian (not necessarily a subgroup of $\mathbf{R}$ ), $f$ is bilinear and $f\left(N^{\prime}, N^{\prime}\right)$ generates $N$, then $G$ is a central extension of $N$.

5. Central extensions and bilinear mappings. Throughout this section assume that $N$ is in the center of $G$. Thus $G$ is determined by the o-group $N^{\prime}$, the abelian $o$-group $N$, and the factor mapping $f: N^{\prime} \times N^{\prime} \rightarrow N$ that satisfies

$$
\begin{gathered}
f\left(0, b^{\prime}\right)=f\left(a^{\prime}, 0\right)=0, \text { and } \\
f\left(a^{\prime}+b^{\prime}, c^{\prime}\right)+f\left(a^{\prime}, b^{\prime}\right)=f\left(a^{\prime}, b^{\prime}+c^{\prime}\right)+f\left(b^{\prime}, c^{\prime}\right) .
\end{gathered}
$$

In particular, any central extension of $N$ by $N^{\prime}$ can be ordered. A central extension $H$ of $N$ by $N^{\prime}$ with factor mapping $h$ is equivalent to $G$ (notation $H \sim G)$ if there exists an isomorphism $\alpha$ of $H$ onto $G$ such that $(0, a) \alpha=(0, a)$ and $\left(a^{\prime}, a\right) \alpha \equiv\left(a^{\prime}, a\right)$ modulo $0 \times N$ for all $a$ in $N$ and all $a^{\prime}$ in $N^{\prime}$. If $H$ is ordered in the usual way, then $\alpha$ is an $o$-isomorphism. It is well known that $H \sim G$ if and only if there exists $t: N^{\prime} \rightarrow N$ such that $t(0)=0$ and

$$
f\left(a^{\prime}, b^{\prime}\right)=h\left(a^{\prime}, b^{\prime}\right)-t\left(a^{\prime}+b^{\prime}\right)+t\left(a^{\prime}\right)+t\left(b^{\prime}\right)
$$

for all $a^{\prime}, b^{\prime}$ in $N^{\prime}$. In particular, $G \sim N^{\prime} \oplus N$ if and only if there exists $t: N^{\prime} \rightarrow N$ such that $t(0)=0$ and $f\left(a^{\prime}, b^{\prime}\right)=-t\left(a^{\prime}+b^{\prime}\right)+t\left(a^{\prime}\right)+t\left(b^{\prime}\right)$ for all $a^{\prime}, b^{\prime}$ in $N^{\prime}$. 
It is easy to verify that if $g$ is a bilinear mapping of $N^{\prime} \times N^{\prime}$ onto $N$, then $g$ satisfies (1) and (2). Moreover, such a $g$ exists if and only if we can choose a representative function $r: N^{\prime} \rightarrow G$ such that

$$
r\left(a^{\prime}+b^{\prime}+c^{\prime}\right)=r\left(a^{\prime}+b^{\prime}\right)+r\left(a^{\prime}+c^{\prime}\right)+r\left(b^{\prime}+c^{\prime}\right)-r\left(a^{\prime}\right)-r\left(b^{\prime}\right)-r\left(c^{\prime}\right)
$$

for all $a^{\prime}, b^{\prime}, c^{\prime}$ in $N^{\prime}$. From (2) we have

$$
f\left(a^{\prime}+b^{\prime}, c^{\prime}\right)-f\left(a^{\prime}, c^{\prime}\right)-f\left(b^{\prime}, c^{\prime}\right)=f\left(a^{\prime}, b^{\prime}+c^{\prime}\right)-f\left(a^{\prime}, b^{\prime}\right)-f\left(a^{\prime}, c^{\prime}\right) .
$$

Thus $f$ is bilinear if it is linear in one variable.

Lemma 5.1. Suppose that $f$ is bilinear, then for $a, b$ in $N$ and $a^{\prime}, b^{\prime}, c^{\prime}$ in $N^{\prime}$ we have:

(i) $-f\left(a^{\prime}, b^{\prime}\right)=f\left(-a^{\prime}, b^{\prime}\right)=f\left(a^{\prime},-b^{\prime}\right)$.

(ii) $f\left(a^{\prime}, b^{\prime}\right)=f\left(-a^{\prime},-b^{\prime}\right)$.

(iii) $\left(a^{\prime}, a\right)+\left(b^{\prime}, b\right)-\left(a^{\prime}, a\right)-\left(b^{\prime}, b\right)=\left(a^{\prime}+b^{\prime}-a^{\prime}-b^{\prime}, f\left(a^{\prime}, b^{\prime}\right)-f\left(b^{\prime}, a^{\prime}\right)\right)$.

For $0=f\left(a^{\prime}-a^{\prime}, b^{\prime}\right)=f\left(a^{\prime}, b^{\prime}\right)+f\left(-a^{\prime}, b^{\prime}\right)$. Thus $-f\left(a^{\prime}, b^{\prime}\right)=f\left(-a^{\prime}, b^{\prime}\right)$ and similarly $-f\left(a^{\prime}, b^{\prime}\right)=f\left(a^{\prime},-b^{\prime}\right)$. (ii) is an immediate consequence of (i), and (iii) follows by computing the left hand side.

Let $D(N)$ be the $d$-closure of $N$, and let $H=N^{\prime} \times D(N)$. For $\left(a^{\prime}, a\right)$ and $\left(b^{\prime}, b\right)$ in $H$ define $\left(a^{\prime}, a\right)+\left(b^{\prime}, b\right)=\left(a^{\prime}+b^{\prime}, f\left(a^{\prime}, b^{\prime}\right)+a+b\right)$. Then $H$ is a central extension of $D(N)$ by $N^{\prime}$, and $G$ is a subgroup of $H$. There is a natural extension of the ordering of $G$ to an ordering of $H$. If $G \sim N^{\prime} \oplus N$, then $H \sim N^{\prime} \oplus D(N)$, but the converse is false. For in [2] there is an example where $N^{\prime}=D(N)=R, H \sim N^{\prime} \oplus N$ and $G x N^{\prime} \oplus N$ [2, p. 862].

Theorem 5.1. Suppose that $N^{\prime}$ is abelian and let $H=D\left(N^{\prime}\right) \times D(N)$. Also suppose that for all $a^{\prime}, b^{\prime}$ in $N^{\prime}$ and for all positive integers $n, f$ satisfies

$$
n f\left(a^{\prime}, b^{\prime}\right)=f\left(n a^{\prime}, b^{\prime}\right)=f\left(a^{\prime}, n b^{\prime}\right) .
$$

Then there exists a unique $g: D\left(N^{\prime}\right) \times D\left(N^{\prime}\right) \rightarrow D(N)$ that satisfies (3) and such that $g\left(a^{\prime}, b^{\prime}\right)=f\left(a^{\prime}, b^{\prime}\right)$ for all $a^{\prime}, b^{\prime}$ in $N^{\prime}$. For $(x, y)$ and $(u, v)$ in $H$ define $(x, y)+(u, v)=(x+u, g(x, u)+y+v)$.

(a) $H$ is a central extension of $D(N)$ by $D\left(N^{\prime}\right)$, and $G$ is a subgroup of $H$.

(b) $H$ is $d$-closed.

(c) For each $h$ in $H$ there exists a positive integer $n=n(h)$ such that $n h \in G$.

(d) There exists a unique extension of the ordering of $G$ to an ordering of $H$. $H$ will be called the d-closure of $G$.

Proof. For each pair $x, y$ in $D\left(N^{\prime}\right)$ there exists a positive integer 
$n=n_{x, y}$ such that $n x, n y \in N^{\prime}$, define $g(x, y)=\left(1 / n^{2}\right) f(n x, n y)$. This definition is independent of the particular choice of $n$. For if $m x, m y \in N^{\prime}$, then $m^{2} f(n x, n y)=f(m n x, m n y)=n^{2} f(m x, m y)$. Thus $\left(1 / n^{2}\right) f(n x, n y)=$ $\left(1 / m^{2}\right) f(m x, m y)$. Let $x, y, z \in D\left(N^{\prime}\right)$ and choose a positive integer $n$ such that $n x, n y, n z, n(x+y)$, and $n(y+z)$ belong to $N^{\prime}$. Then

$$
\begin{aligned}
& g(x+y, z)+g(x, y)=\left(1 / n^{2}\right)[f(n x+n y, n z)+f(n x, n z)] \\
& \quad=\left(1 / n^{2}\right)[f(n x, n y+n z)+f(n y, n z)]=g(x, y+z)+g(y, z) .
\end{aligned}
$$

By a similar argument $g$ satisfies (1) and (3). Also if $g^{\prime}$ is any other extension of $f$ to $D\left(N^{\prime}\right) \times D\left(N^{\prime}\right)$ that satisfies (3), then $n^{2} g^{\prime}(x, y)=$ $g^{\prime}(n x, n y)=f(n x, n y)$. Therefore $g^{\prime}(x, y)=\left(1 / n^{2}\right) f(n x, n y)=g(x, y)$ for all $x, y$ in $D\left(N^{\prime}\right)$.

Clearly (a) is satisfied. To prove (b) it suffices to show that $n(x, y)=$ $(a, b)$ has a solution in $H$, where $n$ is a positive integer and $(a, b) \in H$. By induction

$$
n(x, y)=(n x,[(n-1) n / 2] g(x, x)+n y) .
$$

Thus $x=(1 / n) a$ and

$$
y=(1 / n)(b-[(n-1) n / 2] g((1 / n) a,(1 / n) a))
$$

is a solution. Consider $(x, y)$ in $H$, and let $m$ be a positive integer such that $m x \in N^{\prime}$ and $m y \in N$. Then

$$
\begin{aligned}
& 2 m^{2}(x, y)=\left(2 m(m x),\left(2 m^{2}-1\right) m^{2} g(x, x)+2 m(m y)\right) \\
& \quad=\left(2 m(m x),\left(2 m^{2}-1\right) f(m x, m y)+2 m(m y)\right) \in G .
\end{aligned}
$$

Thus (c) is satisfied. The orderings of $N$ and $N^{\prime}$ can be uniquely extended to orderings of $D(N)$ and $D\left(N^{\prime}\right)$. Define $(x, y) \in H$ positive if $x>0$ or $x=0$ and $y>0$. This extends the ordering of $G$ to an ordering of $H$. But for any extension of the order of $G, h \in H$ is positive if and only if $n h$ is positive in $G$, where $n$ is a positive integer such that $n h \in G$. Thus this extension is unique.

REMARK. If $f$ is bilinear or symmetric or skew-symmetric, then so is $g$. By Theorem $3.2[3$, p. 519] there exists an $a$-closed $a$-extension of $H$ with each component $o$-isomorphic to $\mathbf{R}$. Then

Suppose that $f$ is bilinear. Let $x, y, z \in N^{\prime}$ and let $w=x+y-x-y$.

$$
f(w, z)+f(y, z)+f(x, z)=f(w+y+x, z)=f(x+y, z)=f(x, z)+f(y, z) .
$$

Thus $f(w, z)=0$. Similarly $f(z, w)=0$. Therefore $f(c, z)=f(z, c)=0$ for all $z$ in $N^{\prime}$ and all $c$ in the commutator subgroup of $N^{\prime}$.

Lemma 5.2. If $f$ is bilinear and $N^{\prime}$ coincides with its commutator 
group, then $G=N^{\prime} \oplus N$.

Newmann [11] exhibits an o-group that coincides with its commutator group.

Suppose that $2 N=N$ and $f$ is bilinear. Let $p(x, y)=(1 / 2)[f(x, y)+$ $f(y, x)]$ and let $q(x, y)=(1 / 2)[f(x, y)-f(y, x)]$ for all $x, y$ in $N^{\prime}$. Then $p(q)$ is a symmetric (skew-symmetric) bilinear mapping of $N^{\prime} \times N^{\prime}$ into $N$, and $f(x, y)=p(x, y)+q(x, y)$. Moreover, as in matrix theory, this representation is unique.

THEOREM 5.2. If $2 N=N$ and $f$ is bilinear, then $G \sim H$, where $H$ is the central extension of $N$ by $N^{\prime}$ that is determined by the skew-symmetric part $q$ of. If $f$ is symmetric, then $G \sim N^{\prime} \oplus N$. Thus if $G$ is abelian, then $G \sim N^{\prime} \oplus N$.

Proof. For each $x$ in $N^{\prime}$ define $t(x)=(-1 / 2) f(x, x)$. Then

$$
\begin{aligned}
& -t(x+y)+t(x)+t(y)+q(x, y) \\
& \quad=(1 / 2)[f(x+y, x+y)-f(x, x)-f(y, y)+f(x, y)-f(y, x)]=f(x, y) .
\end{aligned}
$$

Thus $G \sim H$. If $f$ is symmetric, then $H=N^{\prime} \oplus N$, and if $G$ is abelian, then $f$ is symmetric.

Suppose that $N$ and $N^{\prime}$ are abelian and that $f$ is bilinear. Then by Theorem 5.1, we can embed $G$ into its $d$-closure $H=D\left(N^{\prime}\right) \times D(N)$. The factor mapping $g$ associated with $H$ is bilinear, and by Theorem 5.2 we may choose $g$ so that it is skew-symmetric and bilinear. Moreover, $s g(x, y)=g(s x, y)=g(x, s y)$ for all $s \in R$ and for all $x, y$ in $D(N)$. For

$$
n g((m / n) x, y)=g(n(m / n) x, y)=g(m x, y)=m g(x, y) .
$$

Thus $(m / n) g(x, y)=g((m / n) x, y)$. Let $\alpha_{1}, \alpha_{2}, \cdots$ be a basis for the rational vector space $D\left(N^{\prime}\right)$ and consider $X=x_{1} \alpha_{s_{1}}+\cdots+x_{m} \alpha_{s_{m}}$ and $Y=y_{1} \alpha_{t_{1}}+$ $\cdots+y_{n} \alpha_{t_{n}}$ in $D\left(N^{\prime}\right)$. Then

$$
g(X, Y)=\sum x_{i} g\left(\alpha_{s_{i}}, \alpha_{t_{j}}\right) y_{j}
$$

Thus $g$ is determined by the skew symmetric matric $A=\left[g\left(\alpha_{i}, \alpha_{j}\right)\right]$ with components in $D(N)$. Conversely any such matric determines a bilinear skew-symmetric factor mapping of $D\left(N^{\prime}\right) \times D\left(N^{\prime}\right)$ into $D(N)$.

THEOREM 5.3. If $N^{\prime}$ is abelian and $f$ is bilinear, then $G$ is a subgroup of its d-closure $H$ and $H$ is completely determined by $N, N^{\prime}$ and a skew symmetric matrix with entries from $D(N)$. The dimension of this matrix is equal to the rank of the vector space $D\left(N^{\prime}\right)$.

If the rank of $D\left(N^{\prime}\right)$ is finite, say $n$, and $D(N)=R$, then by a suitable choice of coordinates for $D\left(N^{\prime}\right)$ we can get a canonical form for $A$. 


$$
A=\left[\begin{array}{rrrrrr}
0 & 1 & 0 & . & . & . \\
-1 & 0 & 1 & . & . & . \\
0 & -1 & 0 & . & . & . \\
. & . & . & . & . & .
\end{array}\right)
$$

Thus $H$ is determined by $n$ and the rank of $A$. For example if $N^{\prime}=$ $R \times R \times R$ and $N=R$, then we have two non-trivial choices for $f$. One of which is

$$
\begin{aligned}
& f\left(\left(x_{1}, x_{2}, x_{3}\right),\left(y_{1}, y_{2}, y_{3}\right)\right) \\
& \quad=\left[x_{1} x_{2} x_{3}\right]\left[\begin{array}{rrr}
0 & 1 & 0 \\
-1 & 0 & 1 \\
0 & -1 & 0
\end{array}\right]\left[\begin{array}{l}
y_{1} \\
y_{2} \\
y_{3}
\end{array}\right]=-x_{2} y_{1}+\left(x_{1}-x_{3}\right) y_{2}+x_{2} y_{3},
\end{aligned}
$$

and the other is obtained by using the cannonical matrix of rank 2 . Thus for any ordering of $N^{\prime}$ we have at least two non-trivial central $o$-extensions of $N$ by $N^{\prime}$.

Lemma 5.3. If $A$ and $B$ are elements of an ordered semigroup $S$ and $A+B<B+A$, then $n A+n B<n(A+B)<n(B+A)<n B+n A$ for all integers $n$ greater than 2.

Proof. If

$$
\begin{aligned}
& A+(n-1) A+(n-1) B+B=n A+n B \geq n(A+B) \\
& \quad=A+(n-1)(B+A)+B,
\end{aligned}
$$

then $(n-1) A+(n-1) B \geq(n-1)(B+A)$. If

$$
\begin{aligned}
& B+(n-1)(A+B)+A=n(B+A) \geq n B+n A \\
& \quad=B+(n-1) B+(n-1) A+A,
\end{aligned}
$$

then $(n-1)(A+B) \geq(n-1) B+(n-1) A$. Thus the lemma follows immediately by induction on $n$.

Theorem 5.4. If $1 \in N^{\prime} \subseteq R$, then $G$ is abelian.

Proof. By a simple induction argument (see [9] p. 265), $f(x, y)=$ $f(y, x)$ for all integers $x$ and $y$. Let $A=\left(a^{\prime}, a\right)$ and $B=\left(b^{\prime}, b\right)$ be elements of $G$. Then since $a^{\prime}$ and $b^{\prime}$ are rational numbers, there exists a positive integer $n$ such that $n A=\left(x^{\prime}, x\right)$ and $n B=\left(y^{\prime}, y\right)$, where $x^{\prime}$ and $y^{\prime}$ are integers.

$$
\begin{aligned}
n A+n B & =\left(x^{\prime}+y^{\prime}, f\left(x^{\prime}, y^{\prime}\right)+x+y\right) \\
& =\left(y^{\prime}+x^{\prime}, f\left(y^{\prime}, x^{\prime}\right)+y+x\right)=n B+n A .
\end{aligned}
$$

Thus by Lemma 5.3 , we have $A+B=B+A$. 
6. o-groups of rank 2. Throughout this section we assume that $N$ and $N^{\prime}$ are subgroups of $\mathbf{R}$. By Theorem 3.5 [3 p. 523] there exists an $a$-closed $a$-extension $H$ of $G$ such that both components are $o$-isomorphic to $\mathbf{R}$. By Theorem 4.1, either $H$ is a central extension of $\mathbf{R}$ or $H$ is a splitting extension of $\mathbf{R}$. A splitting o-extension of $\mathbf{R}$ by $\mathbf{R}$ is determined by a homomorphism of $\mathbf{R}$ into $\mathbf{P}$. If $H$ is a central extension of $\mathbf{R}$ by $\mathbf{R}$ with a bilinear factor mapping, then $H$ is determined by a skew-symmetric real matrix.

If $N^{\prime}$ is cyclic, then $G$ is a splitting extension of $N$. Thus if $N^{\prime}$ is cyclic and $N$ admits no proper o-automorphisms, then $G=N^{\prime} \oplus N$. In particular, if $N^{\prime}=N=I$, then $G=N^{\prime} \oplus N$. In fact, as Loonstra [9] shows, there are only two normal extensions of $I$ by $I$ (not necessarily ordered) For if $H$ is a normal extension of $I$ by $I$, then $H$ splits over I. Thus $H=I \times I$ and $\left(a^{\prime}, a\right)+\left(b^{\prime}, b\right)=\left(a^{\prime}+b^{\prime}, a s\left(b^{\prime}\right)+b\right)$, where $s$ is a homomorphism of $I$ into the multiplicative group $\{1,-1\}$. Either $s(1)=1$ or $s(1)=-1$. If $s(1)=1$, then $s=\theta$ and $H=I \oplus I$. If $s(1)=$ -1 , then $s(2 n)=1$ and $s(2 n+1)=-1$ for all $n \in I$. Thus the addition rule for $H$ is

$$
\begin{aligned}
& (x, y)+(2 m, n)=(x+2 m, y+n) \\
& (x, y)+(2 m+1, n)=(x+2 m+1, n-y) .
\end{aligned}
$$

In this case $H$ can't be ordered because $-(1,0)+(0,1)+(1,0)=-(0,1)$. Thus $(0,1)$ can't be positive or negative.

If $N=N^{\prime}=R$, then $G$ is $o$-isomorphic to $R \oplus R$. For by Lemma 4.1, $G$ is a central extension of $N$ and by Theorem 5.4, $G$ is abelian. Thus $G$ is an abelian $o$-group of rank 2 with both components $o$-isomorphic to $R$. By Hahn's embedding theorem (see [2]) $G$ is $o$-isomorphic to $R \oplus R$.

Example of a non-abelian o-group of rank 2 that is isomorphic to its group of o-automorphims. Let $N=N^{\prime}=\mathbf{R}$. For $a^{\prime}, b^{\prime} \in N^{\prime}$ define $f\left(a^{\prime}, b^{\prime}\right)=$ 0 and $r\left(a^{\prime}\right)=e^{a \prime}$, where $e$ is transcendental. Then $\left(a^{\prime}, a\right)+\left(b^{\prime}, b\right)=$ $\left(a^{\prime}+b^{\prime}, a e^{b \prime}+b\right)$. By the remark at the end of $\S 3$, an $o$-automorphism $\pi$ of $G$ has a representation $\pi=\left[\begin{array}{ll}1 & \beta \\ 0 & C\end{array}\right]$, where $C \in \mathbf{P}$ and $x^{\prime} \beta=1 \beta\left(e^{x \prime}-\right.$ $1) /(e-1)=\beta \sigma\left(e^{x \prime}-1\right)$ for all $x^{\prime} \in N^{\prime}$. The mapping of $\pi$ onto $\left[\begin{array}{ll}1 & \beta \sigma \\ 0 & C\end{array}\right]$ is an isomorphism of $A(G)$ onto the multiplicative group $A=$ $\left\{\left[\begin{array}{ll}1 & B \\ 0 & C\end{array}\right]: B \in \mathbf{R}\right.$ and $\left.C \in \mathbf{P}\right\}$. The mapping of $\left(a^{\prime}, a\right) \in G$ onto $\left[\begin{array}{ll}e^{a \prime} & 0 \\ a & 1\end{array}\right]$ is an isomorphism of $G$ onto the multiplicative group $B=\left\{\left[\begin{array}{ll}x & 0 \\ y & 1\end{array}\right]: x \in \mathbf{P}\right.$ and $y \in \mathbf{R}\}$. The mapping of $\left[\begin{array}{ll}x & 0 \\ y & 1\end{array}\right]$ onto $\left[\begin{array}{ll}x & 0 \\ y & 1\end{array}\right]^{-1}$ is an isomorphism of $A$ onto $B$. Therefore $G$ is isomorphic to $A(G)$. In particular, there exists a nontrivial splitting o-extension of $G$ by $G$. 
We conclude by giving an example of an o-group of rank 2 that is not a central extension nor a splitting extension of its convex subgroup. Let $G$ be the o-group of the last example, and let $H$ be the subgroup of $G$ that is generated by $\{(a, a): a \in R\}$. We have $(-1,-1)+(1,1)=$ $(0,1-e)$. Thus $H$ has rank 2 .

$$
(1,1)+(0,1-e)=(1,2-e) \neq\left(1, e-e^{2}+1\right)=(0,1-e)+(1,1) .
$$

Thus $H$ is not a central extension.

Lemma. If $\left(b^{\prime}, b\right) \in H$, then $b=\sum_{1}^{m} b_{i} e^{c_{i}}$, where $b_{i}, c_{i} \in R$ and $\sum_{1}^{m} b_{i}=b^{\prime}$. For $\left(b^{\prime}, b\right)=P_{1}+P_{2}+\cdots+P_{n}$, where $P_{i}$ or $-P_{i}$ is a generator. A simple induction on $n$ proves the lemma. In particular, $\left(b^{\prime}, 0\right) \in H$ only if $b^{\prime}=0$. It can be shown that $H=\left\{\left(a, \sum a_{i} e^{b_{i}}\right): a, a_{i}, b_{i} \in R\right.$ and $\left.\sum a_{i}=a\right\}$, but we will not need this.

Now suppose (by way of contradiction) that $H$ is a splitting extension of its convex subgroup $C$. Pick a group $K$ of representatives of $H / C$, and let $(1, a)$ be the element in $K$ with first component 1 . $a=\sum{ }_{1}^{j} a_{i} e^{b_{i}}$, where $\sum_{1}^{j} a_{i}=1$. In particular, $a \neq 0$. By the proof of Theorem 4.1

$$
K=\left\{\left(b^{\prime}, a\left(e^{b \prime}-1\right) /(e-1)\right): b^{\prime} \in R\right\} .
$$

Let $d$ be the least common multiple of the denominators of the $a_{i}$ and let $b^{\prime}=1 / p$, where $p$ is a prime and $p>d$. Then $d\left(\sum a_{i} e^{b_{i}}\right)=\sum c_{i} e^{b_{i}}$ has integral coefficients. By the above lemma

$$
\frac{\left(\sum_{1}^{j} c_{i} e^{b_{i}}\right)\left(e^{b \prime}-1\right)}{e-1}=\sum_{1}^{k} e_{i} e^{a_{i}}
$$

where $e_{i}, d_{i} \in R$. Let $q$ be a positive common multiple of $p$ and the denominators of the $b_{i}$ and the $d_{i}$. Then

$$
\frac{\left[\sum_{1}^{j} c_{i}\left(e^{1 / q}\right)^{u_{i}}\right]\left[\left(e^{1 / q}\right)^{v}-1\right]}{\left(e^{1 / q}\right)^{q}-1}=\sum_{1}^{k} e_{i}\left(e^{1 / q}\right)^{w q_{i}}
$$

where $u_{i}, w_{i}, v \in I$. Without loss of generality we may assume that the $u_{i}$ and the $w_{i}$ are positive integers (multiply both sides of (2) by a suitable power of $\left.e^{1 / q}\right) . e^{1 / q}$ is trancendental. Thus (2) is essentially an equality of elements in the simple transcendental field extension $R(X)$ of $R$.

$$
\frac{\left[\sum_{1}^{j} c_{i} X^{u_{i}}\right]\left[X^{v}-1\right]}{X^{q}-1}=\sum_{i}^{k} e_{i} X^{w_{i}}
$$


$b^{\prime}=1 / p=v / q=v / p v$. Thus there exists a positive integer $n$ such that $p^{n}$ divides $q$, but $p^{n}$ does not divide $v$. The cyclotomic polynomial

$$
f(X)=1+X^{p^{n-1}}+X^{2 p^{n-1}}+\cdots+X^{(p-1) p^{n-1}}
$$

is an irreducible factor of $X^{q}-1$, but it does not divide $X^{v}-1$. Therefore $f(X)$ divides $\sum c_{i} X^{u_{i}}$. Thus $\sum c_{i} X^{u_{i}}=f(X) g(X)$, where $g(X)$ is a polynomial with integral coefficients. Now let $X=1$. Then $d=$ $\sum{ }_{1}^{j} c_{i}=f(1) g(1)=p g(1)$. Thus since $p$ and $d$ are positive and $g(1)$ is an integer, $d \geq p$. But this contradicts our choice of $p$.

Note that the example on page 526 of [3] is a splitting extension of $N$ by $N^{\prime}$; and that $\left.\left\{\left(a^{\prime},-1\right): 0 \neq a^{\prime} \in N^{\prime}\right\} \cup\{0,0)\right\}$ is a group of representatives.

\section{REFERENCES}

1. C. G. Chehata, An algebraically simple ordered group, Proc. London Math. Soc. 2 (1952), 183-197.

2. A. H. Clifford, Note on Hahn's theorem on ordered abelian groups, Proc. Amer. Math. Soc. 5 (1954), 860-863.

3. P. Conrad, Extensions of ordered groups, Proc. Amer. Math. Soc. 6 (1955), 516-528.

4. P. Conrad, The group of order preserving automorphisms of an ordered abelian group, Proc. Amer. Math. Soc. 9 (1958), 382-389.

5 . - A, correction and an improvement of a theorem on ordered groups. To appear in Proc. Amer. Math. Soc.

6. J. de Groot, Indecomposable abelian groups, Indag. Math. 19 (1957), 137-145.

7. N. Hughes, The use of bilinear mappings in the classification of groups of class 2, Proc. Amer. Math. Soc. 2 (1951), 742-747.

8. K. Iwasawa, On linearly ordered groups, J. Math. Soc. Japan 1 (1948), 1-9.

9. F. Loonstra, Sur les extensions du groupe additif des entiers rationnels per le même groupe, Indag. Math. 16 (1954), 263-272.

10. - L'extension du groupe ordonné des entiers rationels par le même groupe, Indag. Math. 17 (1955), 41-49.

11. B. H. Neumann, On ordered groups, Amer. J. Math. 71 (1948), 1-18.

12. - and Hanna Neumann, On a class of abelian groups, Arch. Math. 4 (1953), 79-85.

13. R. P. Rich, Thesis (unpublished) The John Hopkins University (1950).

14. O. Schreier, Über die Erweiterung von Gruppen, Teil I, Monatsh. Math. Phys. 34 (1926), 165-180.

15. A. A. Vinogradov, On the free product of ordered groups, Math. Sb. N. S. 25 (1949), 163-168.

16. H. Yamabe, A condition for an abelian group to be a free abelian group with a finite basis, Proc. Japan Akad. 27 (1951), 205-207.

The Tulane University of Louisiana 



\section{PACIFIC JOURNAL OF MATHEMATICS}

\section{EDITORS}

\section{David Gilbarg}

Stanford University

Stanford, California

R. A. Beaumont

University of Washington

Seattle 5 , Washington
A. L. Whiteman

University of Southern California

Los Angeles 7, California

L. J. PAIGE

University of California

Los Angeles 24, California

\section{ASSOCIATE EDITORS}
E. F. BECKENBACH
C. E. BURGESS
E. HEWITT
A. HORN

\author{
V. GANAPATHY IYER \\ R. D. JAMES \\ M. S. KNEBELMAN \\ L. NACHBIN
}
I. NIVEN
E. G. STRAUS
T. G. OSTROM
H. L. ROYDEN
G. SZEKERES
M. M. SCHIFFER
F. WOLF
K. YOSIDA

\section{SUPPORTING INSTITUTIONS}

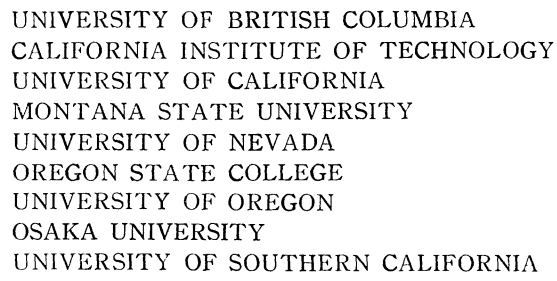

UNIVERSITY OF BRITISH COLUMBIA CALIFORNIA INSTITUTE OF TECHNOLOGY UNIVERSITY OF CALIFORNIA MONTANA STATE UNIVERSITY UNIVERSITY OF NEVADA OREGON STATE COLLEGE UNIVERSITY OF OREGON OSAKA UNIVERSITY UNIVERSITY OF SOUTHERN CALIFORNIA

STANFORD UNIVERSITY

UNIVERSITY OF TOKYO

UNIVERSITY OF UTAH

WASHINGTON STATE COLLEGE

UNIVERSITY OF WASHINGTON

AMERICAN MATHEMATICAL SOCIETY CALIFORNIA RESEARCH CORPORATION HUGHES AIRCRAFT COMPANY SPACE TECHNOLOGY LABORATORIES

Printed in Japan by Kokusai Bunken Insatsusha (International Academic Printing Co., Ltd.), Tokyo, Japan 


\section{Pacific Journal of Mathematics}

\section{Vol. 9, No. 1 \\ May, 1959}

Julius Rubin Blum and Murray Rosenblatt, On the structure of infinitely

divisible distributions . ............................. 1

Robert Geroge Buschman, Asymptotic expressions for

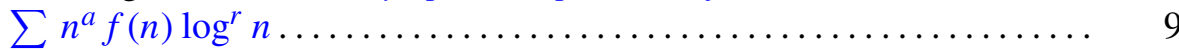

Eckford Cohen, A class of residue systems $(\bmod r)$ and related arithmetical

functions. I. A generalization of Möbius inversion .............. 13

Paul F. Conrad, Non-abelian ordered groups ................... 25

Richard Henry Crowell, On the van Kampen theorem............... 43

Irving Leonard Glicksberg, Convolution semigroups of measures ........ 51

Seymour Goldberg, Linear operators and their conjugates ............ 69

Olof Hanner, Mean play of sums of positional games .............. 81

Erhard Heinz, On one-to-one harmonic mappings ................ 101

John Rolfe Isbell, On finite-dimensional uniform spaces . . ........... 107

Erwin Kreyszig and John Todd, On the radius of univalence of the function

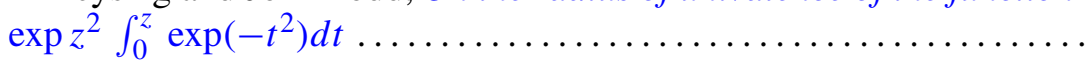

Roger Conant Lyndon, An interpolation theorem in the predicate

calculus......................................... 129

Roger Conant Lyndon, Properties preserved under homomorphism ........ 143

Roger Conant Lyndon, Properties preserved in subdirect products ....... 155

Robert Osserman, A lemma on analytic curves ................ 165

R. S. Phillips, On a theorem due to Sz.-Nagy..................... 169

Richard Scott Pierce, A generalization of atomic Boolean algebras ....... 175

J. B. Roberts, Analytic continuation of meromorphic functions in valued fields................................. 183

Walter Rudin, Idempotent measures on Abelian groups ................ 195

M. Schiffer, Fredholm eigen values of multiply-connected domains ........ 211

V. N. Singh, A note on the computation of Alder's polynomials ......... 271

Maurice Sion, On integration of 1-forms ...................... 277

Elbert A. Walker, Subdirect sums and infinite Abelian groups........... 287

John W. Woll, Homogeneous stochastic processes . .................. 293 